\title{
Process of hydraulic models calibration
}

\author{
Izabela Zimoch ${ }^{1, *}$, and Ewelina Bartkiewicz ${ }^{1}$ \\ ${ }^{1}$ Silesian University of Technology, Institute of Water and Wastewater Engineering, 44-100 Gliwice, 18a Konarskiego St., Poland
}

\begin{abstract}
Mathematical modelling of the water supply systems (WSS) and water quality changes in the system is a complex and difficult task to solve, it requires an interdisciplinary approach to considering the determinants of WSS work. Prognosis models of the WSS in relation to hydraulic quantities are well known and there are many packages that implement these models. These packages allow you to calculate the flow and pressure in the water distribution system under certain operating conditions. However, to make a hydraulic model a useful tool in the management of water supply systems, a calibration process is required. This process involves estimating model parameters to minimize the difference between model results and actual observations. This is a complex and multi-stage process where the network graph and parameters such as roughness coefficient, pump characteristics, or nodal demands are checked and corrected. The following work contains a complex process of calibration of the actual WSS that supplies water to the customers of the selected part of the Silesian agglomeration.
\end{abstract}

\section{Introduction}

A popular trend among water supply companies is the introduction of software to WSS modelling. There are many commercial software that are used to build a model and carry out simulations, such as EPANET, PICCOLO, Mike Net, Water GEMS, and ISYDYW. Increasingly, this software also enables the integration between GIS (Geographic In- formation System), SCADA (Supervisory Control And Data Acquisition) and CIS (Customer Information System) databases, which makes it easier to work on building a WSSs model. Mathematical models reflect operational work of the WSS, thus allowing the analysis of various events occurring in the water distribution subsystem without interfering with real objects. They also support making decisions such as: modernization or extension of water distribution network. Thus, the accuracy of the model must be at a high level. Creating a model is an complex process requiring knowledge of the structure and characteristics of operational work of WSS, and also in the field of modelling. The best solution for such task is its division into individual stages [1]. This approach works well for any modelling project. The most important stages of this process include: data collecting, data verification, testing the model, model calibration and validation, starts analyzes.

During creating hydraulic models, errors may occur, mainly related to the structure and geometry of the network, therefore all stages are closely related to each other creating a loop. If the model reflects the work of WSS, this loop is closed. Fig. 1 shows the action course during the process of creating a hydraulic

\footnotetext{
Corresponding author: izabela.zimoch@polsl.pl
}

model. The calibration process plays a main role in these activities, which in fact enables the creation of a hydraulic model and the launching of simulations.

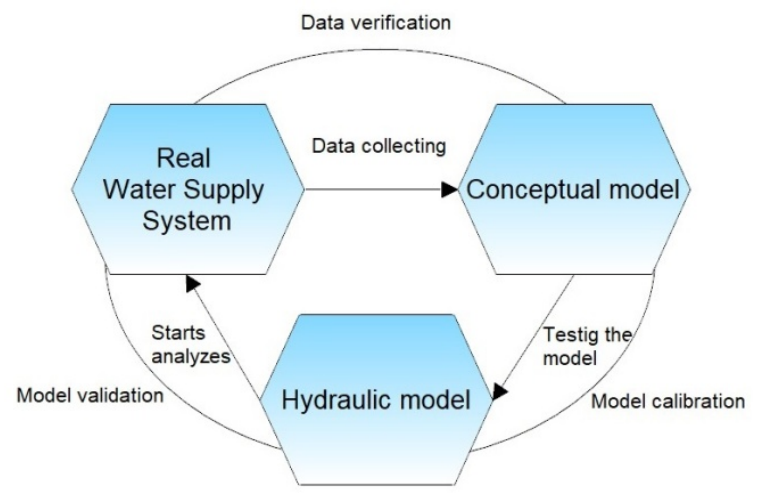

Fig. 1. Diagram of the procedure for creating a hydraulic model.

Calibration is extremely the most difficult and the most important part of modelling, consisting in determining the physical and operational characteristics of the considered WSS. The final stage is to estimate the consistency of the results obtained in the model with the actual field observations. In the case of a large discrepancy between the considered values, the attributes of the objects are again determined until the desired compatibility of the tested parameters is obtained. Calibration, like modelling, can be divided into stages. Seven main steps of the calibration process were determined [1-4]:

a) defining the use of a hydraulic model,

b) initial estimation of the model parameters (conceptual model), 
c) collecting calibration data,

d) preliminary assessment of the model's results,

e) macro-calibration,

f) evaluation of simulation parameters after macrocalibration,

g) micro-calibration.

The model can be used to develop various development strategies of the water supply system, simulation of various operational event scenarios, so the first step in the calibration is to specify exactly what the model should be used for. Walski [5] emphasizes that a once calibrated model can't be used for all purposes.

The second step of the calibration is to reconstruct the geometry of the network together with the water supply infrastructure and preliminary estimation of its parameters. The source of network geometry information is numerous databases, which often contain errors or are incomplete. A quick replenishment of these deficiencies is often impossible due to the lack of design documentation or as-built documentation. In these circumstances, the verification of data is time-consuming and requires geodetic field measurements. Currently, water supply companies use in management from GIS systems, which in the case of integration with a hydraulic model, significantly shorten the process of building a network graph. Most properties of objects (diameters of pipes, tanks, hydraulic characteristics of pumps, reducers' settings) included in the model will have a small degree of uncertainty, however one parameter is characterized by high variability, it is the coefficient of pipe roughness [6-10]. Preliminary values of the coefficient of roughness can be taken from the literature. Many researchers and manufacturers [11-13] of pipes have developed tables with the average size of this parameter, taking into account the material, diameter and age of the pipe. However, ultimately this coefficient should be taken directly from field studies.

Another important element of the calibration is a set of calibration data. According to Walski [14], these data can be divided into three categories: "good", "bad" and "useless". "Good" data is defined as a set of information that is collected during the period when the pressure drop in the network is large enough to draw important conclusions from the calibration. "Bad", on the other hand, contain errors due to incorrect measurement of pressure, improper determination of the height of the measuring device or lack of information about which pumps were activated during data collection. And "useless" data is accumulated when the pressure drops and flow velocities are so small that they are within the range of measurement errors. "Bad" data can be rejected immediately, but how can the two other types be distinguished from each other? The author emphasizes that "good" data is collected when the pressure drop in a given period is greater than the measurement error of devices, while the latter fall within these ranges. Unfortunately, during normal operation, especially the oversized WSS, the water flow rates are so small that the pressure drop is small, so most of the data can be incorrectly classified as "useless". However, these data can be used in the model for checking water levels in tanks, adjusting regulators or setting up pumps.

After process of data collection and verification, macro- and micro-calibration can be started. Macrocalibration is the first proper step in the calibration process, during which larger discrepancies (often above $30 \%)$ are identified between simulation results and actual data [1]. The reasons for these differences are often related to the export of data from the GIS database, i.e. incorrect pipes attributes, wrong network geometry, incorrect settings of valves and pumps. The only way to resolve these errors is to check the data associated with the network graph. Micro-calibration is the final step of this process, in which pipes roughness and water demands are corrected. This stage can be time-consuming, especially with extensive networks, so it can be divided into two parts: static and quasi dynamic calibration. In the first model parameters are adjusted to pressure and flow rate in connection with steady-state observations, while in dynamic calibration model parameters are adapted to various pressure and flow rates, as well as water level in tanks and flow rates at pump stations.

Referring to the Chaplain [2,3,15], static calibration can be divided into three categories:

1) iterative models (trial and error methods),

2) explicit models (models of hydraulic simulations),

3) implicit models (optimization models).

In the first group, unknown parameters are updated at each step/iteration using pressures to solve nonlinear energy continuity equations [6]:

$$
G_{L, i}=H_{i, u}-H_{i, d}-\Delta H_{i}\left(Q_{i}\right)=0
$$

where:

$\mathrm{H}_{\mathrm{i}, \mathrm{u}}$ - pressure head on the i-th section in the upper node $[\mathrm{m}]$;

$\mathrm{H}_{\mathrm{i}, \mathrm{d}}$ - pressure head on the $\mathrm{i}$-th section in the lower node $[\mathrm{m}]$;

$\Delta \mathrm{H}_{\mathrm{i}}$ - pressure head change along the section depending on the type of connection $[\mathrm{m}]$;

$\mathrm{Q}_{i}$ - flow intensity in the i-th section $\left[\mathrm{m}^{3} / \mathrm{h}\right]$;

and flows described by linear mass balance equations [6]:

$$
G_{J, i}=\sum Q_{k}+Q_{d e m, i}=0
$$

where:

$\mathrm{Q}_{\mathrm{k}}$ - flow rate in the k-th pipes $\left[\mathrm{m}^{3} / \mathrm{h}\right]$;

$\mathrm{Q}_{\text {dem, } \mathrm{i}}$-water demand in the node $\mathrm{i}\left[\mathrm{m}^{3} / \mathrm{h}\right]$.

This method requires network graph simplification and is used for small WSS. The second group of models is based on the solution of a wider set of flow continuity equations, using the data obtained from measuring devices. It should be remembered that the number of determined parameters must be equal the number of measurements, and the errors related to the accuracy of measurements are omitted (assuming that all pressure and flows are good). In the last group of implicit models problems are formulated and solved as 
optimization problems consisting in minimizing differences between calculated and actual parameters [16].

\section{Characteristics of water supply system}

The subject of the research is a selected area of the real WSS, supplying water to several urban-industrial agglomerations. This subsystem is supplied by two Water Treatment Plants (WTP "A" and WTP "C"), water well "B" and Pumping Station (PS) "G". The subsystem contains three complexes of Storage Tanks (ST "D", ST "E" and ST "F") with a total capacity of 157 thousand $\mathrm{m}^{3}$ (Fig. 2).

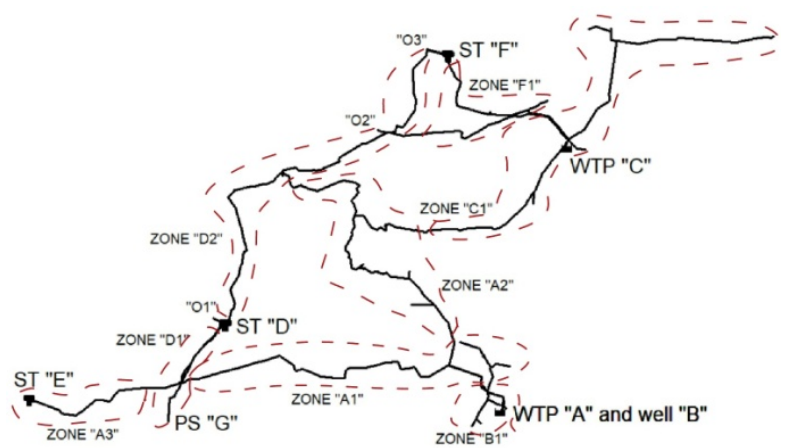

Fig. 2. Scheme of the WSS zone.

In this area there are nodal water demands and three water outflows to another subsystem. On the subsystems map the water outflows are marked with letter "O". Analyzed network is $130 \mathrm{~km}$ long with pipes diameters from $79 \mathrm{~mm}$ to $1600 \mathrm{~mm}$, and it is made of steel, PE-SDR17 polyethylene and cast iron. The oldest pipelines come from 1929 (steel pipes), and the latest from 2016 (PE-SDR17).

The central point of this subsystem is ST "D" (Fig. 2), to which water flows from two objects (WTP "A" and PS "G") and distribute water in two or three directions (depending on the pressure system). In this case ST "E" are water recipient. WTP "A" (average water production $-39,000 \mathrm{~m}^{3} / \mathrm{d}$ ) supplies water to the largest area (areas "A1" and "A2"). Well "B" located near SUW "A" captures an average of $725 \mathrm{~m}^{3} / \mathrm{d}$ to provide water to residents of nearby area "B1". Reservoirs "F" are supplied from the direction "D2" and distribute water in zone "F1". SUW "C" (average water production $-8000 \mathrm{~m}^{3} / \mathrm{d}$ ) due to the reduced water production supplies a small area "C1".

\section{Calibration of the water supply system model}

The model was developed within the framework of NCBiR project POIG.01.03.01-14-034/12. The EPANET 2.0 software was used for the simulation. The calibration process was carried out for data (water production and water demands) from one month (October 2016). Water Distribution System graph was exported from the GIS database, and water demand data was taken from available billing databases. This hydraulic model consists of 871 pipes, 1148 junctions, 302 valves, 11 pumps, 10 tanks and 2 reservoirs. The diurnal curves were determined based on real data collected from the SCADA system for the considered period. In the next step this model was calibrated.

In the first step of model calibration was selection of calibration data. For this purpose were used the filters that reject incorrect measurements of pressure and flow derived from the measurement system online. Four data filters were used during export:

- 1 filter skips measurements marked as unreliable by the network explorer,

- 2 filter verifies the data set in terms of the lack of measurements in a given period, if there are fewer than two measurements in a given hour, data is rejected,

- 3 filter verifies data characterized by lack of variability in the analyzed time interval, then the maximum and minimum values are taken into account for the whole period, if these values are the same, then the filter skips them,

- 4 filter refers to measurements characterized by too large variability of measurements, for which a standard deviation is calculated for each hour, if its value exceeds $5 \%$ for pressures and $10 \%$ for flows, then these values are rejected.

Fig. 3 presents examples of results of using measurement filters. The first digit ( 85 for flows, 325 for pressures) means the number of measuring points, while the values in brackets are as follows: OK number of measurements accepted for the calculation, LIST - number of measurements rejected due to the filters described above, NO DATA - number of measurements without a specified value. The last line is the number of all measurements for each point (from several dozen to several hundred measurements per day for one measuring point) and the number of rejected (incorrect) values.

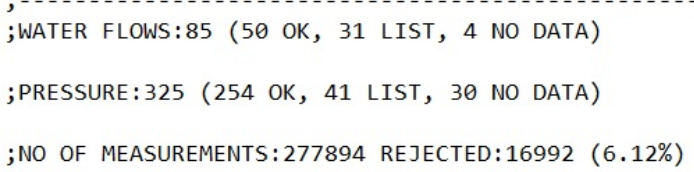

Fig. 3. Example of measurements filters results.

The next stage of the work was the analysis of simulation results. 43 measurement points were tested. Result correlation for the pressure average values was at the level of $99.8 \%$, and flows $98.4 \%$. Despite the results of obtaining a high correlation for average values, the model unfortunately did not reflect the actual work of the network. In these circumstances, three problem areas/objects were identified.

The first object with simulation problem was WT "D", in which the trajectory of tanks filling did not correspond to the measurements (Fig. 4). The reason for this behaviour is the lack of bidirectional flow in the zone "D1" This condition was caused by the failure control of the throttle at reservoirs "G". To improve the 
calculations, a six-chamber tank was replaced with one with a substitute diameter of $66 \mathrm{~m}$ (Fig. 5).

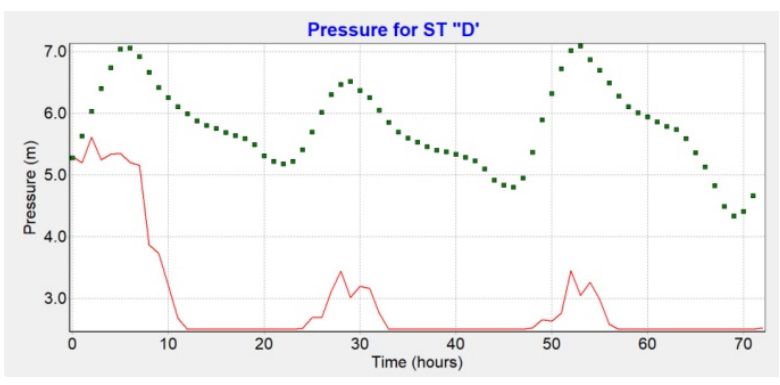

Fig. 4.Trajectory of filling tanks "D" before identifying causes of incompatibility.

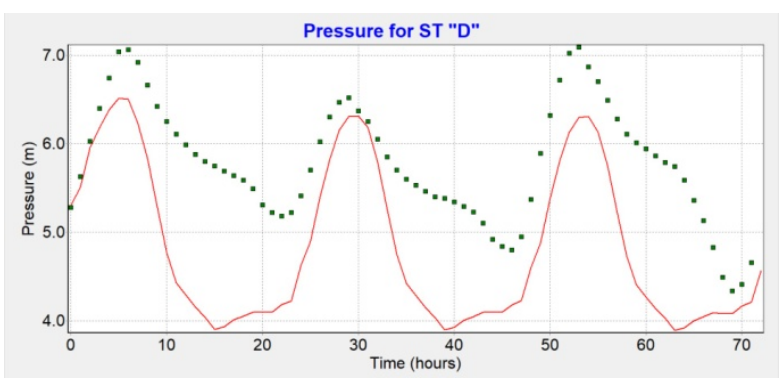

Fig. 5. Trajectory of filling tanks "D" after calibration.

The second case in which the simulation did not correspond to measurements, is zone "A2" supplied by WTP "A" (Fig. 6). The water balance showed that in zone "A2" are discrepancies of water flows of $400 \mathrm{~m}^{3} / \mathrm{h}$ between simulation results and field measurements. After the analysis of zones "A2" and "D2" it was found that on the border of these areas there is a closed valve, reducing the water inflow from the area "D2" (Fig. 7).

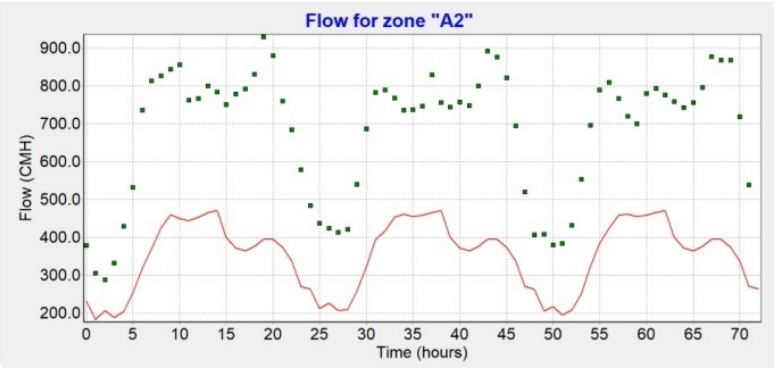

Fig. 6. The water flow in the "A2" zone before identifying causes of incompatibility.

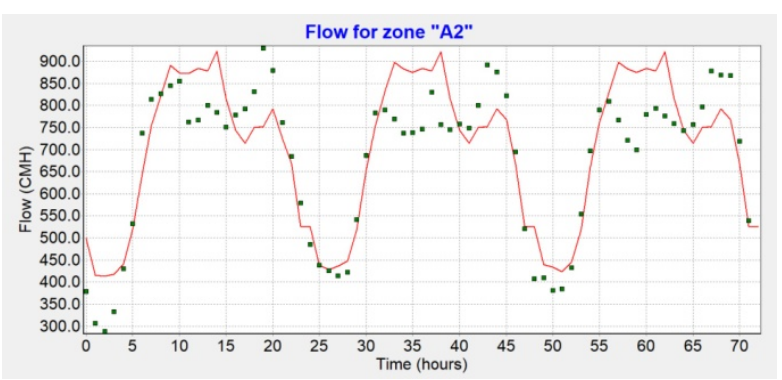

Fig. 7. The water flow in the "A2" zone after calibration.

\section{Research results and conclusions}

Creating hydraulic models is a complex, multi-stage process during which all elements of the water supply network are verified. Today's IT systems enable the integration of databases and computational programs, thus supporting model building and simulation in real time. However, it should be remembered that an extremely important element of modelling is the calibration process. This process requires not only knowledge in the field of model creation and calibration, but also in the scope of the analyzed network.

Despite the good results of the first simulation, the hydraulic model did not reflect the actual work of the WSS. After analyzing a given zone in problematic areas, it was possible to obtain better results.

The next stage of calibration will be microcalibration. As part of this work, it will be necessary to determine the pipes roughness coefficients. The data will include data of age and material of pipelines. This will allow to determine the actual/substitute roughness coefficients. This is a very time-consuming task, considering the size of the analyzed system, therefore this step can be solved using the optimization options. In the next stage of micro-calibration verification should be water demands (placements and value) as well as daily water demands patterns.

After carrying out the micro-calibration and validation, the model will become a useful tool to assist in deciding whether to use or modernize the network.

This work was supported withinstatutory fundsBKM554/RIE-4/2017 and project no BK-286/RIE-4/2017.

\section{References}

1. Modelling Special Interest Group, Water Distribution Modelling Guidelines, (Water New Zealand, 2009)

2. T.M. Walski, J. Water Resour. Plann. Manage. 109, 360 (1983)

3. D.A. Savic, Z. Kapelan, P.M.R. Jonkergouw, Urban Water J. 6, 3 (2009)

4. I. Zimoch, Environ. Prot. Ochr. Sr 30, 3 (2008) http://www.os.not.pl/docs/czasopismo/2008/Zimoc h_3-2008.pdf

5. T. Walski, Proc. AWWA, (1995)

6. L.E. Ormsbee, S. Lingireddy, Water distribution systems handbook, (The McGraw-Hill Companies, New York, 2000)

7. M. Kutylowska, Period. Polytech-Civ. 61, 1 (2017)

8. A. Musz, B. Kowalska, M. Widomski, Ecol. Chem. Eng. S 16, (2009)

9. M. Kutylowska, M. Orlowska-Szostak, Water Pract. Tech. 11, 1 (2016)

10. M. Bożym, I. Kłosok-Bazan, M. Wzorek, Pol. J. Environ. Stud. 27, 3 (2018)

11. Polish Design Standard PN-76/M-34034 
12. Z. Siwoń, J. Cieżak, S. Bogaczewicz, Ochr. Sr. 4, (1998)

13. Fusiotherm company catalog (15.03.2017)

14. T. Walski, J. Am. Water Works Assn. 92, (2000)

15. Z.S. Kapelan, D.A. Savic, G.A. Walters, J. Water Resour. Plann. Manage. 129, (2003)

16. I. Zimoch, E. Łobos, Environ. Protect. Eng. 38, 3 (2012) 